\title{
Enfoques de aprendizaje, autorregulación y autoeficacia y su influencia en el rendimiento académico en estudiantes universitarios de Psicología
}

\author{
Javier Gómez Martínez y Agustín Romero Medina \\ Universidad de Murcia (España)
}

Se analiza la influencia de los enfoques de aprendizaje, los estilos de autorregulación que considera el estudiante en su tarea académica (autorregulación antes, durante y después de la tarea) y la autoeficacia académica percibida de estudiantes universitarios en su rendimiento académico. Se dispone para ello de 136 participantes de $2^{\circ}$ curso del Grado en Psicología. Los resultados muestran que los estudiantes con un buen rendimiento previo, mayor autoeficacia y autorregulación explican mejor el éxito académico. De los enfoques de aprendizaje cabe destacar que, como en otras investigaciones, el más relacionado con el rendimiento académico es el enfoque estratégico. Sin embargo, los estudiantes de menor éxito parecen contradecir los resultados de otras investigaciones predominando en ellos un enfoque profundo. Junto a estos resultados también se muestran relaciones significativas entre enfoque estratégico, autoeficacia y autorregulación, pero no con el enfoque profundo. Se discute la relación bidireccional de estas variables con el rendimiento académico y entre sí, particularmente en lo que se refiere a los enfoques estratégicos y la autorregulación, incluido aquí el factor de metacognición declarativa de autoeficacia y su influencia como expectativa de rendimiento académico.

Palabras clave: Expectativas de éxito, enfoques de aprendizaje, autorregulación, rendimiento académico, estudiantes universitarios de Psicología.

Approaches to learning, self-regulation and self-efficacy in the academic performance of university students. The influence of learning approaches, the styles of self-regulation that the student considers in his academic task (self-regulation before, during and after the task) and the perceived academic self-efficacy of university students in their academic performance are analyzed. For this purpose, 136 participants of the $2^{\text {nd }}$ year of the Degree in Psychology are available. The results show that students with good previous performance, greater self-efficacy and self-regulation better explain academic success. Of the approaches of learning it is necessary to emphasize that, as in other investigations, the most related to the academic performance is the strategic approach. However, the less successful students seem to contradict the results of other investigations predominating in them a deep approach. Along with these results, significant relationships are also shown between strategic approach, self-efficacy and self-regulation, but not with the deep approach. The bidirectional relationship of these variables with academic performance and with each other is discussed, particularly in regard to strategic approaches and self-regulation, including here the declarative metacognition factor of self-efficacy and its influence as an expectation of academic performance

Keywords: Success expectations, learning approaches, self-regulation, academic performance, university students of psychology.

Correspondencia: Agustín Romero Medina. Universidad de Murcia. Facultad de Psicología. Campus de Espinardo. C.P.: 30100. Murcia (España). E-mail: agustinr@um.es 
La investigación actual centrada en las capacidades de autorregulación y en la utilización de determinados enfoques de aprendizaje por parte del estudiante, puede ayudar a controlar mejor, desde la gestión académica, la organización de las metodologías docentes y evaluadoras para lograr los objetivos de rendimiento académico acordes con el Plan Bolonia.

Los enfoques de aprendizaje se refieren al modo o estrategia general para abordar el estudio. Desde los estudios pioneros de Marton y Saljö (1976), Entwistle (1988) y Biggs (1988), se han postulado en el alumnado dos grandes enfoques de aprendizaje antagónicos: profundo (comprender el significado del texto) y superficial (memorizarlo literalmente para aprobar). El primero está mediado por motivaciones intrínsecas (deseo de aprender) y el segundo extrínsecas (evitar fracaso, aprobar asignaturas). Además del enfoque profundo y superficial, tanto Biggs (1988) como Entwistle (1988) sugieren un tercer enfoque de aprendizaje, el estratégico o de logro, en el que la intención del estudiante es aprender y a la vez buscar la mejor calificación posible a través de una planificación adecuada de las variables que intervienen en la calificación académica.

Diversos autores (Biggs, 1987; Marton, Hounsell, y Entwistle, 1984; Valle, González-Cabanach, Núñez, Suárez, Piñeiro, y Rodríguez, 2000), encontraron que los estudiantes con predominio del enfoque profundo tenían mayores expectativas de logro y mejor rendimiento académico que los estudiantes que adoptan un enfoque superficial.

Investigaciones recientes han incluido más variables tales como la autorregulación y la autoeficacia. En cuanto a la autorregulación, Panadero y Tapia (2014), partiendo de las teorías de Zimmerman (2000) y Pintrich (2000), afirman que consiste en el control que ejercen los estudiantes sobre su cognición, comportamiento, emociones y motivación mediante el uso de estrategias para conseguir sus metas de aprendizaje. En diversas investigaciones (Alegre, 2014; Schunck y Zimmerman, 1994) se ha confirmado que en estudiantes con una alta autorregulación también se dan mejores calificaciones, con lo cual es muy probable, según García (2012), que mejore así su percepción de autoeficacia.

Bandura, uno de los investigadores más importantes en el terreno sociocognitivo, influyó en la teoría de la autorregulación de Zimmerman, proponiendo la inclusión de la autoeficacia como uno más de los procesos autorregulatorios, tal y como se ha comprobado en estudios metaanalíticos posteriores (Sitzman y Ely, 2011), en los que se considera la autoeficacia como uno de los componentes valorativos autorregulatorios (junto con la autoevaluación y las atribuciones) que se activan sobre todo en los momentos finales de las tareas de aprendizaje. Bandura (1977) definió la autoeficacia como el conjunto de autopercepciones que tienen las personas acerca de lo que logran con sus capacidades. Un alto nivel de autoeficacia facilita el control autorregulatorio y por tanto es buen predictor de alto rendimiento (Alegre, 2014; Schunck y Zimmerman, 1994) junto con otras variables de la autorregulación; a la inversa, una percepción de autoeficacia baja 
perjudicará el rendimiento académico. En esta perspectiva, Prieto (2003) considera que para una realización exitosa de una tarea no es suficiente con hacerla, sino que es igualmente importante sentirse capacitado para afrontarla y manejar las emociones resultantes. También se ha detectado relación entre tiempo dedicado al estudio y autoeficacia (Valle, Núñez, Cabanach, Rodríguez, González-Pienda, y Rosario, 2009), lo cual también lleva al aumento de la autorregulación y mejora de rendimiento académico; por el contrario, alumnos menos autorregulados y con peor rendimiento académico tenderán a una percepción de menor autoeficacia e incluso al abandono ante los obstáculos (Schunk y Zimmerman, 2008; Zimmerman, 2000, citado en Valle et al., 2009).

Así pues, autoeficacia, autorregulación y enfoques de aprendizaje son en conjunto buenos predictores de alto rendimiento, como se evidencia en la investigación de Pérez, Valenzuela, Díaz, González-Pienda y Núñez (2013), en la cual, en una muestra de 400 estudiantes de carreras diferentes se hallaron relaciones directas y significativas entre alto rendimiento y alta autoeficacia, autorregulación en el estudio y un enfoque de aprendizaje profundo.

\section{Objetivos}

En esta investigación empírica no-experimental de corte descriptivo y correlacional se analizará en población universitaria de primeros cursos de Psicología, la relación de los distintos enfoques de aprendizaje, la autorregulación y la autoeficacia entre sí y según los distintos niveles de rendimiento académico.

\section{MÉTODO}

\section{Participantes}

Participaron en total 136 estudiantes (edad: Media=21.15 años, D.T.=5.47; mujeres: $n=96$ (70.6\%), hombres: $n=40$ (29.4\%) de $2^{\circ}$ curso del Grado de Psicología de la Universidad de Murcia (durante el curso 2014/15).

\section{Instrumentos}

Se utilizaron los siguientes cuestionarios:

Approaches and Study Skills Inventory for Students (ASSIST). En su versión reducida, fue elaborado por Tait, Entwistle y McCune (1998). Se utilizó la versión traducida al español por Hidalgo et al. (2009). Es un autoinforme que evalúa la autopercepción del uso de estrategias, habilidades y enfoques de aprendizaje en entorno académico. El ASSIST consta de 18 ítems con respuesta tipo Likert que oscila desde 1=Totalmente en desacuerdo a 5=Totalmente de acuerdo. Los 18 ítems están clasificados en tres factores: enfoque profundo (Factor I), enfoque superficial (Factor II), enfoque estratégico (Factor III). En el estudio de validación de la versión española de Hidalgo et 
al. (2009) se encontraron niveles adecuados de fiabilidad mediante el coeficiente alfa de Cronbach para los tres factores (Factor $\mathrm{I}=0.74$, Factor $\mathrm{II}=0.68$, Factor $\mathrm{III}=0.65$ ).

Cuestionario de Autoeficacia Académica. Este cuestionario fue elaborado por el segundo de los autores y está formado por 10 ítems de escala valorativa sumatoria que oscila de 0 a 10 que evalúan diferentes cualidades relacionadas con el contexto académico (esfuerzo, constancia, intensidad para estudiar en los exámenes, comprensión, memoria fotográfica, asociación, control, concentración, conocer los requisitos para aprobar y aprender en profundidad). No está constatada su validez interna, pero tras calcular el correspondiente coeficiente alfa de Cronbach presenta un coeficiente de 0.731 y un 0.844 en elementos estandarizados.

Cuestionario de Autorregulación en el estudio. Fue elaborado por el segundo de los autores y consta de 22 ítems agrupados en cuatro fases de la tarea de estudio (antes de empezar a estudiar, mientras estudia, después de estudiar y tras la nota del examen) que se responden con diferentes escalas de medida (tipo Likert y dicotómicas). El cuestionario trata de medir la capacidad de autorregulación del estudiante en las diferentes fases de estudio y en el total. De este cuestionario se calculó el coeficiente alfa de Cronbach (alfa=0.607, siendo una fiabilidad aceptable pero baja) y también se le aplicó el modelo de Rasch (Abellán-Pérez, 2018), pudiendo comprobar que los ítems se ajustan a los principios de dicho modelo.

\section{Procedimiento y análisis de datos}

La aplicación de la prueba a los sujetos corrió a cargo de un psicólogo entrenado y se hizo en el horario habitual de clases. Se contó con la autorización institucional y del profesor a cargo del curso. Los estudiantes cumplimentaron posteriormente un archivo Word con todos los cuestionarios y preguntas, así como el consentimiento informado donde se detallaba el objetivo de la investigación y se comunicaba el carácter voluntario, anónimo y sin retribución de la participación. Dicho archivo ya cumplimentado lo subieron a la plataforma web docente de la Universidad de Murcia ("Aula virtual"); posteriormente los datos fueron registrados con Excel y luego analizados mediante el paquete estadístico SPSS v. 24. Por la realización de la investigación fueron recompensados con bonos canjeables por décimas en la nota final de asignaturas del Grado.

Para el tratamiento estadístico de la variable rendimiento académico se tuvo en cuenta la nota media de las asignaturas cursadas hasta el momento de la investigación (final de primer cuatrimestre de $2^{\circ}$ curso), es decir, las notas de primer curso de Grado en Psicología. Se clasificó a los sujetos en cuatro grupos: (1) suspenso, con notas comprendidas entre 0 y 4.99 (n=3), (2) aprobado, entre 5 y 6.5 ( $n=51)$, (3) notable, entre 6.51 y $7.99(n=64)$ y (4) sobresaliente entre 8 y $10(n=18)$; se hizo entre 8 y 10 por la escasa frecuencia de notas entre 9 y 10. 


\section{RESULTADOS}

Enfoques de aprendizaje, autorregulación y autoeficacia según niveles de rendimiento académico

Las puntuaciones en enfoques de aprendizaje difieren significativamente entre los sujetos según su nivel de rendimiento académico. Esto ocurre en el enfoque estratégico $\left(F(3.110)=3.323, \quad p=.023, \operatorname{Eta}^{2}=.338\right.$; ver figura 1) y en el enfoque profundo $\left(F(3.100)=3.818, p=.012, \mathrm{Eta}^{2}=.143\right.$; ver figura 2$)$, en sendos ANOVAs unifactoriales. Sin embargo, el enfoque superficial no muestra puntuaciones significativas en relación con el rendimiento académico, según los datos del cuestionario ASSIST.

Figura 1. Puntuación en enfoque estratégico en cada categoría del rendimiento académico en el Grado de Psicología

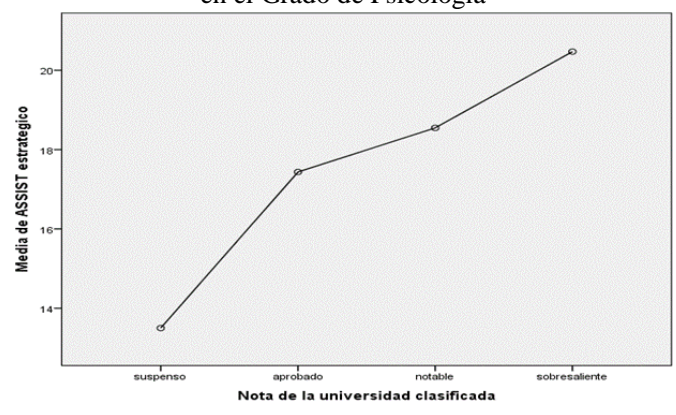

En la figura 1 vemos que, a mayor puntuación en el enfoque estratégico, el rendimiento también aumenta.

En cambio, en la figura 2, a pesar de encontrar resultados significativos en el enfoque profundo, como demuestra el ANOVA, los estudiantes suspensos puntuaron alto en enfoque profundo. No obstante, la muestra de sujetos suspensos es muy baja, solo tres, por lo que debemos tener precaución con estos resultados.

Figura 2. Puntuación en enfoque profundo en cada categoría del rendimiento académico en el Grado de Psicología

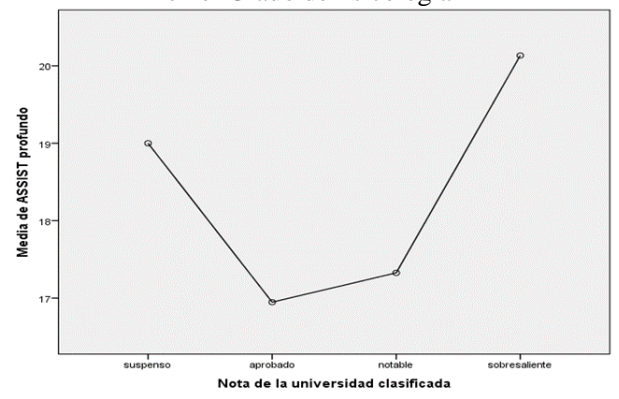


Por último, en el enfoque superficial, aunque al realizar el ANOVA no se encontrasen resultados significativos, la figura 3 muestra una tendencia a disminuir el uso del enfoque superficial conforme aumenta la puntuación. Estos datos parecen manifestar que los alumnos de suspenso, aprobado y notable tienden a buscar el mínimo esfuerzo para conseguir el objetivo de aprobar la materia.

Figura 3. Puntuación en enfoque superficial en cada categoría del rendimiento académico en el Grado de Psicología

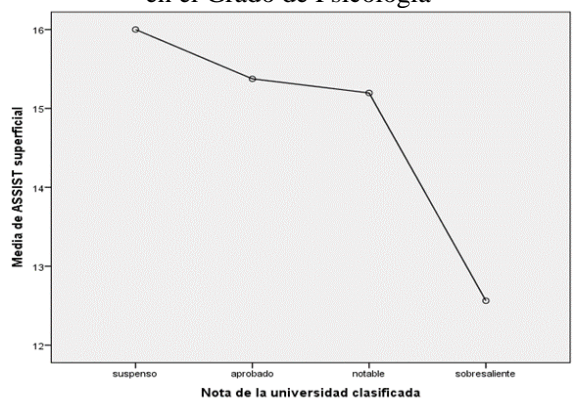

Así pues, enfoque profundo y estratégico se relacionan con alto rendimiento académico. Esto se corrobora con el análisis post hoc de Tukey, en el cual las diferencias de medias entre los estudiantes de Sobresaliente con los de otras categorías de notas son significativas, tanto en el enfoque profundo (diferencias con los estudiantes de notable y aprobado) como en el estratégico (diferencias con los estudiantes de aprobado), tal y como se observa en la figura 4, siendo la combinación de puntuaciones altas en enfoque profundo y estratégico, las más utilizadas por los estudiantes con mejor rendimiento.

Figura 4. Distribución de las medias marginales estimadas en los enfoques (ASSIST) en cada categoría del rendimiento

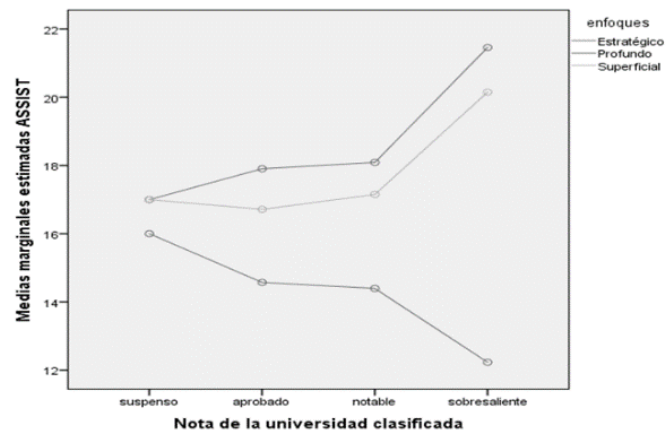


En autorregulación del estudio encontramos diferencias significativas según rendimiento académico, tanto en la p como en el alto tamaño del efecto $(F(3.132)=5.428$, $p=.001, E t a^{2}=.617$; ver figura 5).

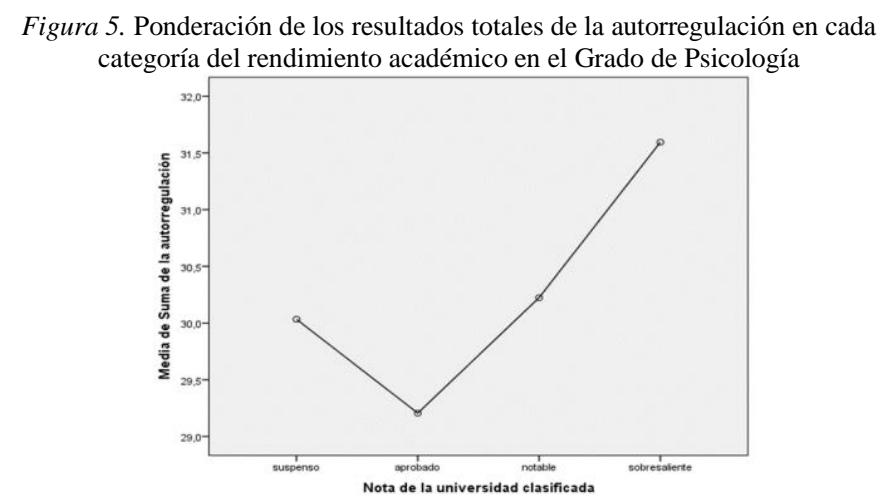

Los estudiantes con mejor nota parecen ser más autorregulados. La anomalía de que los estudiantes suspensos parecen ser más autorregulados que los aprobados no tiene significación estadística (el post hoc de Tukey entre suspensos y aprobados no da significación de diferencias de medias, cosa que sí ocurre entre aprobados y sobresalientes, con una diferencia de medias de $2.38, p=.001$ ) y por otra parte, la escasa muestra de estudiantes suspensos (tres sujetos), tampoco permitiría ir más allá.

Los resultados en puntuaciones de autoeficacia académica percibida en función de los cuatro niveles de rendimiento académico muestran diferencias significativas $\left(F(3.132)=5.762, p=.001, E t a^{2}=.489\right)$ con un patrón similar al obtenido con puntuaciones en enfoque estratégico (ver figura 1), por lo que se puede asumir que un buen nivel en la autoeficacia que perciben los estudiantes sobre ellos mismos puede influir en el rendimiento académico.

\section{Relación entre todas las variables y modelo predictivo de rendimiento académico}

En la tabla 1 vemos las correlaciones biseriales entre todas las variables analizadas. Tanto la autoeficacia percibida por los estudiantes como el enfoque estratégico correlacionan positivamente con todas las variables, a excepción de la nota de selectividad. Sin embargo, con el enfoque superficial presenta una correlación negativa, indicando que sujetos con predominio en un enfoque superficial muestran puntuaciones más bajas en autoeficacia académica y menor predominancia de un enfoque estratégico. La variable autorregulación correlaciona con el rendimiento académico anterior y actual 
(nota de selectividad y de universidad), con el enfoque estratégico y con la autoeficacia académica. Por lo que respecta al enfoque superficial se encuentran correlaciones negativas con todas las variables, por tanto, es evidente que no es un buen enfoque de aprendizaje en educación superior.

Tabla 1. Correlación entre variables influyentes en el rendimiento académico (ASSIST)

\begin{tabular}{lcccccc}
\hline & $\begin{array}{c}\text { Autoeficacia } \\
\text { académica }\end{array}$ & Autorregulación & $\begin{array}{c}\text { ASSIST } \\
\text { estratégico }\end{array}$ & $\begin{array}{c}\text { ASSIST } \\
\text { superficial }\end{array}$ & $\begin{array}{c}\text { ASSIST } \\
\text { profundo }\end{array}$ & $\begin{array}{c}\text { Nota } \\
\text { universidad }\end{array}$ \\
\hline Autoeficacia académica & 1 & & & & & \\
\hline Autorregulación & $.320^{* *}$ & 1 & & & & \\
\hline ASSIST estratégico & $.738^{* *}$ & $.469^{* *}$ & 1 & & & \\
\hline ASSIST superficial & $-.384^{* *}$ & -.112 & $-.321^{* *}$ & 1 & & 1 \\
\hline ASSIST profundo & $.380^{* *}$ & .129 & $.239^{*}$ & -.182 & 1 & $.303^{* *}$ \\
\hline Nota universidad & $.399^{* *}$ & $.405^{* *}$ & $.339^{* *}$ & $-.231^{*}$ & .100 & $.379^{* *}$ \\
\hline Nota de selectividad & .150 & $.197^{*}$ & .183 & $-.207^{*}$ & &
\end{tabular}

El enfoque profundo correlaciona con el enfoque estratégico (no son excluyentes), con la autoeficacia y el rendimiento académico universitario. Es destacable que no correlacione de manera significativa con la autorregulación, confirmando que esta variable está en la órbita del enfoque estratégico del estudio, en el que se intenta controlar en lo máximo posible el ambiente y el proceso de estudio.

En lo que se refiere al rendimiento académico anterior, en este caso la selectividad, correlaciona con las calificaciones universitarias, aunque en lo que respecta a los enfoques solo correlaciona negativamente con el enfoque superficial.

En resumen, el rendimiento académico universitario vemos que muestra correlaciones significativas $(p<.01)$ con autorregulación, autoeficacia, selectividad, enfoque estratégico y profundo. En cambio, con el enfoque superficial correlaciona negativamente.

En lo referente a determinar un modelo predictivo de estas variables con el rendimiento académico, tras un análisis mediante un modelo de regresión lineal de todas las variables, se muestra el siguiente modelo (véase la tabla 2).

Tabla 2. Modelo de análisis de regresión lineal predictivo de rendimiento académico universitario

\begin{tabular}{|c|c|c|c|c|c|}
\hline Modelo & $\mathrm{R}$ & $\mathrm{R}^{2}$ & $\mathrm{R}$ ajustado & Cambio R ${ }^{2}$ & Sig. F \\
\hline Autorregulación & $.405^{\mathrm{a}}$ & .164 & .157 & .164 & .000 \\
\hline Autorregulación, Nota de selectividad & $.507^{\mathrm{b}}$ & .257 & .246 & .093 & .000 \\
\hline Autorregulación, Nota de selectividad, Autoeficacia & $.569^{\mathrm{c}}$ & .323 & .308 & .066 & .000 \\
\hline
\end{tabular}

La tabla 2 muestra un modelo que explica un total del $30.8 \%$ de la varianza total del rendimiento académico, siendo una puntuación aceptable en un ámbito como es el educativo. Las variables por orden de peso en el modelo son: autorregulación (15.7\%), nota de selectividad (8.9\%) y autoeficacia (6.2\%). Este modelo no incluye ningún enfoque 
de aprendizaje, pudiendo deberse a la alta correlación que tiene con las diversas variables del modelo, produciéndose un problema de colinealidad.

\section{DISCUSIÓN Y CONCLUSIONES}

Así pues, los estudiantes con mejores puntuaciones mayoritariamente presentan enfoque estratégico y también enfoque profundo. Otros estudios, tales como los de Valle et al. (2000), Marton et al. (1984) y Biggs (1987), encontraron resultados similares a los nuestros, aunque no remarcaron la importancia en el enfoque estratégico y lo englobaron como una variable más de cada enfoque. La coincidencia, que también se observa en Barrón y Llimpe (2017), radica en que, con puntuaciones altas, tanto en la escala de enfoque profundo como en las correspondientes subescalas de estrategia y motivación, los sujetos mostraban mejores resultados en cuanto a rendimiento académico. También nuestros datos coinciden con los conseguidos en dos investigaciones de diseño preexperimental pretest-postest en estudiantes de primer año de universidad (Gargallo y Campos, 2013; Rosário, Mourão, Núñez, González-Pienda, Solano, y Valle, 2007), en las que se evidenciaron que los estudiantes consiguieron mejoras tanto en sus notas, como un aumento en el uso de los enfoques estratégico y profundo, además de una disminución del uso del enfoque superficial. Junto a estos datos, las mismas investigaciones promovieron la enseñanza de tareas para mejorar la autorregulación, consiguiendo mejoras en ella.

En definitiva, ambos enfoques no son excluyentes sino que, más bien, a la hora de abordar el proceso de aprendizaje, una combinación de ambos sería más conveniente para la consecución de buenos resultados que utilizar uno solo. No obstante, el enfoque que correlaciona en mayor medida con el rendimiento académico es el enfoque estratégico, puesto que un estudiante que sabe cómo utilizar sus estrategias y cuáles son los requisitos para conseguir mejores puntuaciones, además de tener una motivación tanto de logro como de aprender, proporcionará como resultado un alto rendimiento.

En relación con los estudiantes de bajo rendimiento, estos obtuvieron puntuaciones altas en enfoque profundo, lo que nos lleva a pensar que adoptar un enfoque profundo sin un plan estratégico en ocasiones puede llevar al fracaso.

El enfoque estratégico correlaciona de forma significativa con la autorregulación, ya que un alumno que utiliza un enfoque estratégico controla su proceso de estudio, así como las horas de estudio, cómo abordar el temario, el modo en que planifica dependiendo del objetivo, la evaluación o el temario, por lo que también será más autorregulado cumpliendo el plan estratégico que realizó para su proceso de estudio.

La variable autorregulación ya hemos comprobado que también influye en el rendimiento académico. Estos resultados en autorregulación se asemejan a los encontrados en otros trabajos (Alegre, 2014; Fernández et al.,, 2013; Fernández, García, y González, 2013; Rosário, Núñez, y González-Pienda, 2004; Schunck y Zimmerman, 1994; 
Zimmerman, 2000), con la excepción de la puntuación obtenida por los sujetos suspensos que puede ser debido al escaso tamaño muestral.

Así, los estudiantes con recursos estratégicos, capaces de regular los mecanismos de su motivación, cognición y conducta, además de otros aspectos como la planificación para la consecución de objetivos y que adopten un buen enfoque de aprendizaje (combinación de enfoque estratégico y profundo), podrán conseguir mejor rendimiento, tanto en el ámbito académico como profesional.

Como era de esperar, también se corrobora una alta correlación entre autorregulación y autoeficacia académica (véase tabla 1), lo cual confirma la presencia de la autoeficacia como un elemento de los procesos de autorregulación (Sitzman y Ely, 2011). Otros autores en sus trabajos ya mencionaron este mismo hecho (Pajares, 2001; Zimmerman y Martínez-Pons, 1990), en el que es crucial que los niveles de autoeficacia y la capacidad de autorregulación sean adecuados, puesto que son procesos motivacionales que impulsan al estudiante a sentirse competente y confiado en sus propias habilidades, consiguiendo estudiantes motivados, competentes y responsables de su proceso de aprendizaje.

Por otra parte, estudiantes con autopercepción de óptima autoeficacia obtendrán mejores calificaciones, siguiendo un patrón ascendente de autoeficacia en las diferentes clasificaciones del rendimiento. Otras investigaciones confirman la misma hipótesis que nosotros encontramos en nuestra investigación (Alegre, 2014; Fernández et al., 2013; Pajares, 2001; Schunck y Zimmerman, 1994), incluso con otros instrumentos de evaluación para el mismo constructo.

Al relacionar entre sí las variables de enfoques, autorregulación, autoeficacia y rendimiento académico (Tabla 1), la mayor correlación se dio entre la variable autoeficacia y el enfoque estratégico, seguido de una alta correlación también entre la autoeficacia y el enfoque profundo. Estas correlaciones explicarían cómo los estudiantes construyen mejor su autoeficacia combinando ambos enfoques para lograr un buen rendimiento, y a su vez, estas experiencias de éxito reforzarían la autoeficacia que tienen sobre sus capacidades, puesto que el predominio de estos enfoques implica ser conscientes de las estrategias y capacidades que poseen para superar con éxito las diversas tareas que realizan.

Enfoque estratégico, autorregulación y autoeficacia son las variables que presentan mayor tamaño del efecto $(.338, .489, .617$, respectivamente; o sea, la fuerza o magnitud que tienen sobre la variable de forma independiente a las demás). Como además estas tres variables tienen fuertes correlaciones entre ellas, podríamos pensar que están englobadas en un mismo factor probablemente autorregulatorio, y además muy importante en este ámbito educativo universitario. En esta misma línea iría el modelo predictivo sobre rendimiento académico en el análisis de regresión, de no ser porque no aparece el enfoque estratégico; esto puede ser debido por un problema de colinealidad al existir fuertes correlaciones del enfoque estratégico con otras variables (autorregulación y autoeficacia). 
Pese a este problema, tres de nuestras cuatro variables que planteamos en nuestra hipótesis entran dentro de este modelo que explicó un $30.8 \%$ de la varianza del rendimiento académico, siendo la autorregulación la que explicó un mayor porcentaje de la varianza del rendimiento académico, con un $15.7 \%$, siendo un porcentaje notable para una sola variable, puesto que una variable como el rendimiento académico cuenta con multitud de factores que influyen entre sí. Las siguientes variables con mayor porcentaje fue la nota de selectividad con un $8.9 \%$ y la autoeficacia con un $6.2 \%$. La nota de selectividad parece pues tener importancia en el modelo predictivo, dado que se trata de una de las primeras grandes pruebas por las que pasa un alumno. Si esta experiencia es superada con éxito, reforzará su autoeficacia, teniendo una autopercepción de sus capacidades adecuadas a su nivel. Estas variables ayudarán al alumno a seguir pautas más autorreguladoras pues una vez que conoce sus capacidades, organizará y planificará su proceso de estudio en base a ellas.

En futuras investigaciones se debería ampliar la muestra de estudiantes con bajo rendimiento académico (una de las limitaciones de este estudio) o incluir estudiantes de otro tipo de titulaciones, así como considerar el diseño de investigaciones experimentales pretest-postest, instruyendo a cada grupo en uno de los enfoques.

A modo de conclusión, en esta investigación se demostró una relación entre la combinación de enfoques estratégico y profundo, con el éxito académico, además de la existencia de una relación entre el uso de un enfoque estratégico con el uso de estrategias autorreguladoras y mayores niveles de autoeficacia. Estas tres variables podrían ser una buena base para una mejora de los procesos de estudio y no hablar de ellas por separado, porque una retroalimenta a la otra, consiguiendo que una mejora en una de ellas ayude a la otra. También debería plantearse desde etapas iniciales de la educación (al menos desde secundaria) trabajar y enseñar a los alumnos los diversos enfoques de aprendizaje y su uso, igualmente, además de enseñarles los beneficios de ser estudiantes autorregulados.

\section{REFERENCIAS}

Abellán, C. (2018). Aplicación del modelo de Rasch en un cuestionario de autorregulación en el estudio. Murcia: Universidad de Murcia, Trabajo Fin de Grado (no publicado).

Alegre, A. (2014). Autoeficacia académica, autorregulación del aprendizaje y rendimiento académico en estudiantes universitarios iniciales. Propósitos y Representaciones, 2(1), $79-120$.

Bandura, A. (1977). Self-efficacy: toward a unifying theory of behavioral change. Psychological Review, 84(2), 191.

Barrón, H.J., y Llimpe, Y. (2017). Enfoques de aprendizaje y rendimiento académico en estudiantes de medicina del primer año de la Universidad Nacional Mayor de San Marcos. Anales de la Facultad de Medicina, 78(1), 49-54.

Biggs, J. (1987). Student approaches to learning and studying. Hawthorn, Victoria: Australian Council for Educational Research. 
Biggs, J. (1988). Approaches to learning and to essay writing. In R. Schmeck (Ed.), Learning strategies and learning styles (pp. 185-228). New York: Plenum Press.

Entwistle, N.J. (1988). Motivational factors in students' approaches to learning. En R. Schmeck (Ed.), Learning strategies and learning styles (pp. 21-55). New York: Plenum Press.

Fernández, E., Bernardo, A., Suárez, N., Cerezo, R., Núñez, J.C., y Rosário, P. (2013). Predicción del uso de estrategias de autorregulación en educación superior. Anales de Psicología, 29(3), 865-875.

Fernández, M.S., García, T., y González, P. (2013). Autorregulación y rendimiento académico en matemáticas. Aula Abierta, 41(1), 39-48.

García, M. (2012). La autorregulación académica como variable explicativa de los procesos de aprendizaje universitario. Profesorado. Revista de Currículum y Formación del Profesorado, 16(1), 203-221.

Gargallo, B., y Campos, C. (2013). Aprendizaje estratégico en la universidad. Una materia para aprender a aprender. In XI Jornadas de Redes de Investigación en Docencia Universitaria: Retos de futuro en la enseñanza superior: Docencia e investigación para alcanzar la excelencia académica (pp. 2185-2196). Alicante: Universidad de Alicante, Instituto de Ciencias de la Educación.

Hidalgo, M.D., Romero, A., González, F., García-Sevilla, J., Pérez, M.A., Carrillo, ... Peñaranda, M. (2009). Herramientas de evaluación de los estilos de aprendizaje en universitarios: Traducción y adaptación del cuestionario ASSIST. Comunicación presentada a las IV Jornadas sobre el Espacio Europeo de Educación Superior: "El Espacio Europeo de Educación Superior en el horizonte del 2010". Murcia, 22, 23 y 24 de septiembre de 2009.

Marton, F., y Säljö, R. (1976). On qualitative differences in learning. I. Outcome and process. British Journal of Educational Psychology, 46, 4-11.

Marton, F., Hounsell, D.J., y Entwistle, N.J. (1984). The experience of learning. Edinburgh: Scottish Academic Press.

Pajares, F. (2001). Self-efficacy beliefs in academic settings. Review of Educational Research, 66(4), 543-578.

Panadero, E., y Tapia, J. (2014). How do students self-regulate? Review of Zimmerman's cyclical model of self-regulated learning. Anales de Psicología, 30(2), 450-462.

Pérez, M.V., Valenzuela Castellanos, M., Díaz, A., González-Pienda, J.A., y Núñez, J.C. (2013). Dificultades de aprendizaje en estudiantes universitarios de primer año. Atenea (Concepción), (508), 135-150.

Pintrich, P.R. (2000). The role of goal orientation in self-regulated learning. En M. Boekaerts, P.R. Pintrich \& M. Zeidner (Eds.), Handbook of Self-Regulation (pp. 452-502). San Diego, CA: Academic Press.

Prieto, L. (2003). La autoeficacia en el contexto académico. Exploración bibliográfica comentada. Atlanta, Georgia: Emory University.

Rosário, P., Núñez, J., y González-Pienda, J. (2004). Stories that show how to study and how to learn: an experience in Portuguese school system. Electronic Journal of Research in Educational Psychology, 1, 131-144.

Rosário, P., Mourão, R., Núñez, J.C., González-Pienda, J.A., Solano, P., y Valle, A. (2007). Eficacia de un programa instruccional para la mejora de procesos y estrategias de aprendizaje en la enseñanza superior. Psicothema, 19(3), 353-358.

Schunk, D.H., y Zimmerman, B. (1994). Self-regulation of learning and performance: issues and educational applications. Mahwah, NJ: Erlbaum.

Sitzmann, T., y Ely, K. (2011). A Meta-Analysis of Self-Regulated Learning in Work-Related Training and Educational Attainment: What we know and where we need to go. Psychological Bulletin, 137(3), 421-442. 
Tait, H., Entwistle, N.J., y McCune, V. (1998). Assist a reconceptualization of the Approaches to Studying Inventory. In C. Rust (Ed.), Improving students as learners (pp. 262-271). Oxford: Oxford Bookes University.

Valle, A., González-Cabanach, R., Núñez, J.C., Suárez, P., Piñeiro, I., y Rodríguez, S. (2000). Enfoques de aprendizaje en estudiantes universitarios. Psicothema, 12, 368-375.

Valle, A., Nuñez, J.C., Cabanach, R.G., Rodriguez, S., Gonzalez-Pienda, J.A., y Rosario, P. (2009). Perfiles motivacionales en estudiantes de secundaria: Análisis diferencial en estrategias cognitivas, estrategias de autorregulación y rendimiento académico. Revista Mexicana de Psicología, 26(1), 113-124.

Zimmerman, B.J. (2000). Attaining self-regulation: a social cognitive perspective. In M. Boekaerts, P.R. Pintrich \& M. Zeidner (Eds.), Handbook of Self-Regulation (pp. 13-40). San Diego, CA: Academic Press.

Zimmerman, B.J., y Martínez-Pons, M. (1990). Student differences in selfregulated learning: Realting grade, sex, and giftedness to self-efficaccy and strategy use. Journal of Educational Psychology, 82, 51-59.

Recibido: 19 de diciembre de 2018 Recepción modificaciones: 26 de enero de 2019 Aceptado: 27 de enero de 2019 